\title{
DECOMPOSIÇÃO DA INTERAÇÃO TRIPLA SIGNIFICATIVA UTILIZANDO O COMANDO CONTRAST DO PROC GLM DO SAS APLICADO AO MODELO DE CLASSIFICAÇÃO TRIPLA PARA DADOS BALANCEADOS ${ }^{(1)}$
}

\author{
MARIA CRISTINA STOLF NOGUEIRA ${ }^{(2)}$; JOSÉ EDUARDO CORRENTE ${ }^{(2)}$
}

\begin{abstract}
RESUMO
Na análise de experimentos em classificação tripla com interação para dados balanceados, onde os fatores envolvidos podem ter níveis qualitativos ou quantitativos e a interação é significativa, é relevante a obtenção da decomposição dos graus de liberdade da interação e dos graus de liberdade dos efeitos envolvidos. No que se refere à interação tripla significativa a análise estatística tem, ainda, como objetivo testar o efeito de um dos fatores de tratamentos aninhados, em relação à combinação dos níveis dos outros fatores de tratamentos envolvidos. Através do PROC GLM do SAS é possível testar esse efeito utilizando o comando CONTRAST ou ESTIMATE. Assim, a proposta do presente trabalho é a obtenção das somas de quadrados de tais efeitos utilizando o comando CONTRAST do PROC GLM / SAS. Isso é possível através da construção da hipótese a ser testada e da aplicação do método dos contrastes ortogonais, visando a facilidade na checagem dos resultados obtidos. Para tal, são dados um exemplo numérico e o programa $S A S$.

Palavras-chave: experimentos em classificação tripla, interação, decomposição da interação.
\end{abstract}

\section{ABSTRACT \\ TRIPLE INTERACTION FRACTIONING USING PROC GLM CONTRAST/SAS, APPLIED TO A THREE-WAY LAYOUT FOR BALANCED DATA}

\begin{abstract}
In a three-way analysis of variance, in which significant interaction among the involved factors is detected, it might be important to get the fractioning of the interaction degrees of freedom added to the degrees of freedom of the involved factors. In the case of a significant triple interaction, the effecet of one nested factor is usually checked against the combined effect of the remaining factors. The main aim of this research work has been to get the sum of squares of such effect through the CONTRAST command of PROC GLM, available in the SAS computer program. The feasibility of checking hypothesis and the application of orthogonal contrasts is evaluated through the checking of the obtained results. A numerical example is provided.
\end{abstract}

Key words: three-way layout, interaction, decomposition of interaction.

\section{INTRODUÇÃO}

Os modelos de classificação tripla para dados balanceados têm-se mostrado de grande utilidade na experimentação agronômica. Segundo KuEHL (1994), as comparações entre tratamentos podem ser afetadas substancialmente pelas condições em que ocorrem, e, interpretações claras dos efeitos de um tratamento precisam ser levadas em conta para o efeito de outros tratamentos. Isso significa que a interação entre fatores pode estar presente em tais delineamentos e, sendo a interação significativa, é necessário fazer um estudo dessa interação através de sua decomposição em fatores aninhados. De acordo com HinKELMANN e KEMPTHORNE (1994), o procedimento de variar um

$\left({ }^{1}\right)$ Recebido para a publicação em 24 de maio e aceito em 9 de dezembro de 1999.

$\left({ }^{2}\right)$ Departamento de Ciências Exatas - ESALQ/USP, Caixa Postal 9, 13418-900 Piracicaba (SP). 
fator, por vez, via de regra se aplica quando o objetivo é estabelecer uma lei fundamental, o que conduziria ao conhecimento detalhado do efeito de um fator, quando os outros são mantidos constantes. Nenhuma informação, no entanto, é obtida a respeito da dependência dos efeitos de um fator nos níveis para os quais os outros fatores são mantidos constantes, pelo fato de que os níveis de um fator apresentam comportamento diferenciado na presença do outro fator, e vice-versa. Segundo NogUEIRA (1997), novamente de acordo com HinKELMANN e KEMPTHORNE (1994), outro procedimento experimental seria considerar todas as possíveis combinações de níveis simultaneamente. Isso permitiria obter informação acerca dos fatores principais e, especialmente, da interação entre os vários fatores. Tais idéias e suas aplicações práticas na experimentação agronômica, bem como na experimentação científica, de maneira geral foram introduzidas por FiSHER (1935) e YATES (1937).

Assim, neste trabalho, o estudo da interação significativa é feito através da decomposição dos graus de liberdade da interação adicionados aos graus de liberdade do nível do fator aninhado.

Para a análise de dados segundo esse modelo, com interação significativa utilizando o software $S A S$, o resultado não é imediato, pois requer uma programação com a finalidade de se obter a referida decomposição. Isso, em geral, é feito no PROC GLM utilizando a opção $B Y$ e fixando o fator aninhado, conforme encontrado no manual do SAS/STAT (1990). O problema, nesse caso, consiste na inadequação do teste $\mathrm{F}$ da análise da variância, sendo necessário refazê-lo manualmente aplicando o denominador correto.

O objetivo deste trabalho é mostrar outra forma de utilização do PROC GLM do SAS, juntamente com o comando CONTRAST, na decomposição da interação, ou seja, tal decomposição será encarada como contrastes entre médias para os efeitos aninhados, sendo, dessa forma, o teste $\mathrm{F}$ aplicado adequadamente.

\section{MATERIAL E MÉTODOS}

$\mathrm{O}$ modelo matemático para um experimento de classificação tripla envolvendo os fatores A, B e C com interação, considerando todos os efeitos fixos em uma estrutura inteiramente ao acaso, é dado da seguinte maneira:

$$
\begin{aligned}
& \quad \mathrm{y}_{\mathrm{ijkl}}=\mu+\alpha_{\mathrm{i}}+\beta_{\mathrm{j}}+\delta_{\mathrm{k}}+(\alpha \beta)_{\mathrm{ij}}+(\alpha \delta)_{\mathrm{ik}}+(\beta \delta)_{\mathrm{jk}}+ \\
& +(\alpha \beta \delta)_{\mathrm{ijk}}+\xi_{\mathrm{ijkl}} \\
& \text { para } i=1, I ; j=1,, J ; k=1,, K \mathrm{e} l=1,, L .
\end{aligned}
$$

onde

Yijkl é o valor observado, referente ao nível $i$ do fator A, combinado com o nível $j$ do fator B e com o nível $k$ do fator $C$, na repetição $l$;

$\mu$ é o efeito de média geral;

$\alpha_{\mathrm{i}}$ é o efeito do nível $i$ do fator $\mathrm{A}$, definido como $\boldsymbol{\alpha}_{\mathrm{i}}=\boldsymbol{\mu}_{\mathrm{i} . .}-\boldsymbol{\mu}$, sendo $\mu_{\mathrm{i} . .}$ a média do nível $i$ do fator $\mathrm{A}$;

$\beta_{\mathrm{j}}$ é o efeito do nível $j$ do fator $\mathrm{B}$, definido como

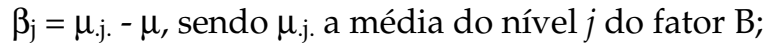

$\delta_{\mathrm{k}}$ é o efeito do nível $k$ do fator $\mathrm{C}$, definido como $\delta_{\mathrm{k}}=\mu_{. . \mathrm{k}}-\mu$, sendo $\mu_{. \mathrm{k}}$ a média do nível $k$ do fator $C$;

$(\alpha \beta)_{\mathrm{ij}}$ é o efeito da interação de $\mathrm{A} \operatorname{com} \mathrm{B}$, definido como $(\alpha \beta)_{i j}=\mu_{\mathrm{ij} .}-\left(\mu-\alpha_{\mathrm{i}}-\beta_{\mathrm{j}}\right)$, sendo $\mu_{\mathrm{ij} .}$ a média do nível $i$ do fator A, combinado com o nível $j$ do fator $\mathrm{B}$;

$(\alpha \delta)_{\text {ik }}$ é o efeito da interação de $\mathrm{A}$ com $\mathrm{C}$, definido como $(\alpha \delta)_{\mathrm{ik}}=\mu_{\mathrm{i} . \mathrm{k}}-\left(\mu-\alpha_{\mathrm{i}}-\delta_{\mathrm{k}}\right)$, sendo $\mu_{\mathrm{i} . \mathrm{k}}$ a média do nível $i$ do fator $\mathrm{A}$, combinado com o nível $k$ do fator C;

$(\beta \delta)_{j \mathrm{k}}$ é o efeito da interação de $\mathrm{B}$ com $\mathrm{C}$, definido como $(\beta \delta)_{j \mathrm{k}}=\mu_{\mathrm{j} \mathrm{k}}-\left(\mu-\beta_{\mathrm{j}}-\delta_{\mathrm{k}}\right)$, sendo $\mu_{\mathrm{j} \mathrm{k}}$ a média do nível $j$ do fator $\mathrm{B}$, combinado com o nível $k$ do fator $\mathrm{C}$;

$(\alpha \beta \delta)_{\mathrm{ijk}}$ é o efeito da interação de $\mathrm{A}, \mathrm{B}$ e C, definido como

$$
(\alpha \beta \delta)_{\mathrm{ijk}}=\mu_{\mathrm{ijk}}-\left(\mu+\alpha_{\mathrm{i}}+\beta_{\mathrm{j}}+\delta_{\mathrm{k}}+(\alpha \beta)_{\mathrm{ij}}+(\alpha \delta)_{\mathrm{ik}}+\right.
$$

$\left.+(\beta \delta)_{\mathrm{jk}}\right)$

sendo $\mu_{\mathrm{ijk}}$ a média do nível $i$ do fator $\mathrm{A}$, combinado com o nível $j$ do fator B e com o nível $k$ do fator $C$;

$\xi_{\mathrm{ijkl}}$ é o erro experimental associado a $\mathrm{y}_{\mathrm{ijkl}} \mathrm{e}$ ambos considerados independentes e identicamente distribuídos, com distribuição $\mathrm{N}\left(0, \sigma^{2}\right)$.

Outra forma de escrever o modelo adotado é:

$$
\mathrm{y}_{\mathrm{ijkl}}=\mu_{\mathrm{ijk}}+\xi_{\mathrm{ijkl}}
$$

$\operatorname{com} \mu_{\mathrm{ijk}}=\mu+\alpha_{\mathrm{i}}+\beta_{\mathrm{j}}+\delta_{\mathrm{k}}+(\alpha \beta)_{\mathrm{ij}}+(\alpha \delta)_{\mathrm{ik}}+\beta \delta_{\mathrm{jk}}+(\alpha \beta \delta)_{\mathrm{ijk}}$, sendo a média da combinação do nível $i$ do fator $\mathrm{A}$ com o nível $j$ do fator B e com o nível $k$ do fator $C$, para $i=1, \ldots, I ; j=1, \ldots, J$ e $k=1, \ldots, K$.

Obtidos os dados e segundo o modelo matemático adotado, a principal hipótese formulada é a referente à interação $\mathrm{A} \times \mathrm{B} \times \mathrm{C}$, seguida das hipóteses referentes às interações $\mathrm{A} \times \mathrm{B}, \mathrm{A} \times \mathrm{C}$ e $\mathrm{B} \times \mathrm{C}$, que são:

$$
\begin{aligned}
& \mathrm{H}_{01}=\mu_{\mathrm{ijk}}-\mu_{\mathrm{ijk}}-\mu_{\mathrm{ij} j^{\prime} \mathrm{k}}-\mu_{\mathrm{i}^{\prime} \mathrm{k}}+\mu_{\mathrm{ij} \mathrm{j}^{\prime} \mathrm{k}^{\prime}}+\mu_{\mathrm{i}^{\prime} \mathrm{k}^{\prime}}+\mu_{\mathrm{i} \mathrm{j}^{\prime} \mathrm{k}}- \\
& -\mu_{i^{\prime} j^{\prime} k^{\prime}}=0 \text {, para todo } i \neq i^{\prime}, j \neq j^{\prime} \text { e } k \neq k^{\prime} \text {; } \\
& \mathrm{H}_{02}=\mu_{\mathrm{ij} .}-\mu_{\mathrm{ij} .}{ }^{\prime}-\mu_{\mathrm{i}^{\prime} \mathrm{j}}+\mu_{\mathrm{i}^{\prime} \mathrm{j}^{\prime} \cdot}=0 \text {, para todo } \mathrm{i} \neq \mathrm{i}^{\prime}, \mathrm{j} \neq \mathrm{j}^{\prime} \\
& \mathrm{H}_{03}=\mu_{\mathrm{i} . \mathrm{k}}-\mu_{\mathrm{i} . \mathrm{k}^{\prime}}-\mu_{\mathrm{i}^{\prime} \cdot \mathrm{k}}+\mu_{\mathrm{i}^{\prime} \cdot \mathrm{k}^{\prime}}=0 \text {, para todo } \mathrm{i} \neq \mathrm{i}^{\prime}, \mathrm{k} \neq \mathrm{k}^{\prime}, \\
& \mathrm{H}_{04}=\mu_{\mathrm{j} \mathrm{k}}-\mu_{\mathrm{j} \mathrm{j}^{\prime} \mathrm{k}}-\mu_{\mathrm{j} \mathrm{k} \mathrm{k}^{\prime}}+\mu_{\mathrm{j} \mathrm{j}^{\prime} \mathrm{k}^{\prime}}=0 \text {, para todo } \mathrm{j} \neq \mathrm{j}^{\prime}, \mathrm{k} \neq \mathrm{k}^{\prime} \text {, }
\end{aligned}
$$


e testadas através da aplicação do teste $\mathrm{F}$, da seguinte maneira:

$$
\frac{\text { QM A x B x C }}{\text { QM Resíduo }} \cap \mathrm{F}[(\mathrm{I}-1)(\mathrm{J}-1)(\mathrm{K}-1),(\mathrm{IJK}-1)(\mathrm{L}-1)],
$$

sob $\mathrm{H}_{01}$.

Se a hipótese $\mathrm{H}_{01}$ for rejeitada, conclui-se que a interação A x B x C é significativa, e, que o fator A tem um efeito diferenciado na presença de cada combinação dos níveis dos fatores $\mathrm{B}$ com $\mathrm{C}$, o mesmo ocorrendo para os efeitos dos fatores $\mathrm{B}$ e C. Nesse caso, a análise dos dados terá continuidade com a decomposição dos graus de liberdade das interações envolvidas, adicionados os graus de liberdade do fator aninhado. A decomposição é feita de acordo com o objetivo da pesquisa.

Supondo-se, por exemplo, que o interesse seja verificar o efeito do fator $\mathrm{A}$, dada cada combinação dos níveis dos fatores $B$ com $C$, isto é, estudar o efeito do fator $\mathrm{A}$ aninhado na combinação do nível $j$ do fator $\mathrm{B}$, com o nível $k$ do fator $\mathrm{C}$, o modelo matemático adotado agora passa a ser o seguinte:

$$
\mathrm{y}_{\mathrm{i}(\mathrm{k} \mathrm{k}) \mathrm{l}}=\mu+\beta_{\mathrm{j}}+\delta_{\mathrm{k}}+(\beta \delta)_{\mathrm{jk}}+\alpha_{\mathrm{I}(\mathrm{jk})}+\xi_{\mathrm{ijk} \mathrm{l}}
$$

para $i=1, \ldots, I ; j=1, \ldots, J ; k=1, \ldots, K$ e $l=1, \ldots, L$. sendo $\alpha_{\mathrm{i}(\mathrm{jk})}$ o efeito do fator $\mathrm{A}$, dado o nível $j$ do fator $\mathrm{B}$, combinado com o nível $k$ do fator $\mathrm{C}$, definido como $\alpha_{\mathrm{i}(\mathrm{jk})}=\alpha_{\mathrm{i}}+\alpha \beta_{\mathrm{ij}}+\delta_{\mathrm{k}}+(\alpha \beta)_{\mathrm{ij}}+(\alpha \delta)_{\mathrm{ik}}+(\alpha \delta)_{\mathrm{ik}}=(\mu)_{\mathrm{i}(\mathrm{jk})}-$ $\mu_{\mathrm{jk}}$, onde $\mu_{\mathrm{i}(\mathrm{jk})}$ é a média do nível $i$ do fator $\mathrm{A}$, dado o nível $j$ do fator $\mathrm{B}$, combinado com o nível $k$ do fator $\mathrm{C}$ o modelo matemático apresentado acima pode ser descrito ainda da seguinte maneira:

$$
\mathrm{y}_{\mathrm{i}(\mathrm{kj}) \mathrm{l}}=\mu_{\mathrm{i}(\mathrm{jk})}+\varepsilon_{\mathrm{ijk} \mathrm{l}}
$$

sendo a hipótese

$\mathrm{H}_{05}: \mu_{1(\mathrm{jk})}=\mu_{2(\mathrm{jk})}=\ldots=\mu_{\mathrm{I}(\mathrm{jk})}$ vs. $\mathrm{H}_{\mathrm{a}}$ : pelo menos

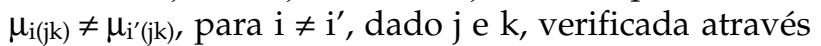
do teste $\mathrm{F}$, da seguinte maneira:

$$
\frac{\mathrm{QM} \mathrm{A}\left(\mathrm{B}_{\mathrm{j}} \mathrm{C}_{\mathrm{k}}\right)}{\mathrm{QM} \mathrm{Resíduo}}=\frac{\frac{\mathrm{SQ} A\left(\mathrm{~B}_{\mathrm{j}} \mathrm{C}_{\mathrm{k}}\right)}{(\mathrm{I}-1)}}{\mathrm{QMResíduo}} \cap \mathrm{F}[(\mathrm{I}-1),(\mathrm{IJK}-1)(\mathrm{L}-1)]
$$

sob $\mathrm{H}_{05}$

A análise dos dados com essa estrutura, através do software SAS, é feita no PROC GLM utilizando opção $B Y$, fixando os fatores $B$ e $C$, conforme encontrado no manual do SAS/STAT (1990). O problema, nesse caso, constitui-se no fato de que o teste $F$, obtido na análise da variância para a hipótese testada, não está calculado adequadamente, sendo necessário refazê-lo manualmente aplicando o denominador correto QM Resíduo com (IJK-1)(L-1) graus de liberdade. Uma alternativa para contornar o problema é a aplicação de contrastes ortogonais, entre médias, para os efeitos aninhados. De acordo com a ilustração dada, considera-se aplicação de contrastes ortogonais entre médias,do fator $\mathrm{A}$, dado o nível $j$ do fator $\mathrm{B}$ combinado com o nível $k$ do fator $C$, cuja hipótese formulada para $h=1, \ldots,(I-1)$, é a seguinte:

$$
\mathrm{H}_{06}: \mathrm{Y}\left(\mathrm{h}_{\mathrm{jk}}\right)=0 \text { vs. } \mathrm{H}_{\mathrm{a} 6}: \mathrm{Y}\left(\mathrm{h}_{\mathrm{jk}}\right) \neq 0 \text {, para } h=1, \ldots,
$$

$(I-1)$,

onde

$$
Y\left(h_{j k}\right)=\sum_{i=1}^{I} c_{h i} \mu_{i(j k)} \quad \text { com } \quad \sum_{i=1}^{I} c_{h i}=0
$$

e

$$
\begin{aligned}
\mu_{\mathrm{i}(\mathrm{jk})}=\mu_{. \mathrm{jk}}+\alpha_{\mathrm{i}(\mathrm{jk})=} & {\left[\mu+\beta_{\mathrm{j}}+\delta_{\mathrm{k}}+(\beta \delta)_{\mathrm{jk}}\right]+} \\
& +\left[\alpha_{\mathrm{i}}+(\alpha \beta)_{\mathrm{ij}}+(\alpha \delta)_{\mathrm{ik}}+(\alpha \beta \delta)_{\mathrm{ijk}}\right]
\end{aligned}
$$

Portanto:

$$
\begin{gathered}
Y\left(h_{j k}\right)=\sum_{i=1}^{I} c_{h i}\left[\beta_{j}+\delta_{k}+(\beta \delta)_{j k}+\alpha_{i j(k)}\right]= \\
{\left[\beta_{j}+\delta_{k}+(\beta \delta)_{j k}\right] \sum_{i=1}^{I} c_{h i}+\sum_{i=1}^{I} c_{h i} \alpha_{i(j k)}} \\
Y\left(h_{j k}\right)=\sum_{i=1}^{I} c_{h i} \alpha_{i j k j)} \\
Y\left(h_{j k}\right)=\sum_{i=1}^{I} c_{h i} \alpha_{i}+\sum_{i=1}^{I} c_{h i}(\alpha \beta)_{i(j)}+\sum_{i=1}^{I} c_{h i}(\alpha \delta)_{i(k)}+ \\
+\sum_{i=1}^{I} c_{h i}(\alpha \beta \delta)_{i(j k)}
\end{gathered}
$$

cujo estimador não-tendencioso é $\hat{Y}\left(h_{j k}\right)$ e $\operatorname{SQY}\left(\mathrm{h}_{\mathrm{j} k}\right)=\frac{\left[\hat{Y}\left(\mathrm{~h}_{\mathrm{j}}\right)\right]^{2}}{\sum_{\mathrm{i}=1}^{1} c_{h i}^{2}}$ com 1 grau de liberdade. Obtém-se, ainda, que $\operatorname{SQA}\left(\mathrm{B}_{\mathrm{j}} \mathrm{C}_{\mathrm{k}}\right)=\sum_{\mathrm{h}=1}^{\mathrm{I}-1} \operatorname{SQY}\left(\mathrm{h}_{\mathrm{j} k}\right) \operatorname{com}(\mathrm{I}-1)$ graus de liberdade.

A hipótese $\mathrm{H}_{06}$ será testada através do teste $\mathrm{F}$, da seguinte maneira:

$$
\frac{\mathrm{QM} \mathrm{Y}\left(\mathrm{h}_{\mathrm{jk}}\right)}{\mathrm{QM} \text { Resíduo }}=\frac{\frac{\mathrm{SQY}\left(\mathrm{h}_{\mathrm{jk}}\right)}{(1)}}{\mathrm{QM} \text { Resíduo }} \cap \mathrm{F}[1,(\mathrm{IJK}-1)(\mathrm{L}-1)]
$$

sob $\mathrm{H}_{06}$. 
A aplicação desse método no software $S A S$ viabiliza-se com a utilização do comando CONTRAST do PROC GLM, cuja sintaxe é dada a seguir:

$$
\begin{aligned}
& \text { CONTRAST "Y }\left(\mathrm{h}_{\mathrm{jk}}\right) \text { " A } \mathrm{Ch}_{\mathrm{h}} \ldots \mathrm{ChI} \mathrm{A}^{*} \mathrm{~B} \mathrm{Ch}_{\mathrm{h}} 1 \ldots \mathrm{chI} \\
& \mathrm{A}^{*} \mathrm{C} \text { Ch1 ... ChI } A^{*} B^{*} \mathrm{CCh}_{\mathrm{C} 1} \ldots \mathrm{ChI}
\end{aligned}
$$

Essa linha de programação $S A S$ fornece, como resultados, a SQY $\left(h_{j}\right)$ com 1 grau de liberdade e ainda o valor calculado do teste $\mathrm{F}$, de acordo com o exposto, e também a PROB > F. Os demais contrastes ortogonais são estruturados dessa mesma maneira.

Assim, considerando os (I-1) contrastes ortogonais, dados um j e um $k$ quaisquer, estruturados na maneira exposta e utilizando o comando CONTRAST com a seguinte sintaxe, teremos:

\begin{tabular}{|c|c|c|c|c|c|c|c|c|c|c|}
\hline \multirow[t]{9}{*}{ "A $\left(\mathrm{B}_{\mathrm{j}} \mathrm{C}_{\mathrm{k}}\right){ }^{\prime \prime}$} & $\mathbf{A}$ & $\mathrm{c}_{11}$ & $\mathrm{C}_{12}$ & $\ldots$ & $\mathrm{c}_{1 \mathrm{I}}$ & $A^{*} B$ & $\mathrm{c}_{11}$ & $\mathrm{C}_{12}$ & $\ldots$ & $\mathrm{c}_{1 \mathrm{I}}$ \\
\hline & $A^{*} C$ & $\mathrm{c}_{11}$ & $\mathrm{c}_{12}$ & $\ldots$ & $\mathrm{c}_{1 \mathrm{I}}$ & $A^{*} B^{*} \mathbf{C}$ & $\mathrm{c}_{11}$ & $\mathrm{c}_{12}$ & $\ldots$ & $\mathrm{c}_{1 \mathrm{I}}$ \\
\hline & $\mathbf{A}$ & $\mathrm{c}_{21}$ & $\mathrm{c}_{22}$ & $\ldots$ & $\mathrm{c}_{2 \mathrm{I}}$ & $A^{*} B$ & $\mathrm{c}_{21}$ & $\mathrm{c}_{22}$ & $\ldots$ & $\mathrm{c}_{2 \mathrm{I}}$ \\
\hline & $A^{*} \mathbf{C}$ & $\mathrm{c}_{21}$ & $\mathrm{c}_{22}$ & $\ldots$ & $\mathrm{c}_{2 \mathrm{I}}$ & $A^{*} B^{*} C$ & $\mathrm{c}_{21}$ & $\mathrm{c}_{22}$ & $\ldots$ & $\mathrm{C}_{2 \mathrm{I}}$ \\
\hline & & $\vdots$ & $\vdots$ & $\vdots$ & $\vdots$ & $\vdots$ & $\vdots$ & $\vdots$ & $\vdots$ & $\vdots$ \\
\hline & $\mathbf{A}$ & $\mathrm{C}_{(\mathrm{I}-1) 1}$ & $\mathrm{C}(\mathrm{I}-1) 2$ & $\cdots$ & & $A * B$ & & & $\cdots$ & $\mathrm{C}(\mathrm{I}-1) \mathrm{I}$ \\
\hline & $A^{*} C$ & $\mathrm{C}(\mathrm{I}-1) 1$ & $\mathrm{C}_{(\mathrm{I}-1) 2}$ & $\ldots$ & $\mathrm{C}(\mathrm{I}-1) \mathrm{I}$ & $A^{*} B^{*} \mathbf{C}$ & $\mathrm{C}_{(\mathrm{I}-1) 1}$ & $\mathrm{C}_{(\mathrm{I}-1) 2}$ & $\ldots$ & $\mathrm{C}_{(\mathrm{I}-1) \mathrm{I}}$ \\
\hline & & $\Downarrow$ & $\Downarrow$ & $\Downarrow$ & $\Downarrow$ & & $\Downarrow$ & $\Downarrow$ & $\Downarrow$ & $\Downarrow$ \\
\hline & & (1) & (2) & $\ldots$ & (I) & & (1) & (2) & $\ldots$ & (I) \\
\hline
\end{tabular}

\section{CONTRAST}

Essas (I-1) linhas de programação SAS, separadas pelas vírgulas, fornecem como resultados a $\operatorname{SQA}\left(\mathrm{B}_{\mathrm{j}} \mathrm{C}_{\mathrm{k}}\right)=\sum_{\mathrm{h}=1}^{\mathrm{I}-1} \mathrm{SQY}\left(\mathrm{h}_{\mathrm{jk}}\right), \operatorname{com}(\mathrm{I}-1)$ graus de liberdade. $\mathrm{O}$ valor do teste $\mathrm{F}$ obtido para testar a hipótese $\mathrm{H}_{06}$, para qualquer $j$ e $k$, está correto e de acordo com o exposto acima.

Observa-se que as colunas representadas por (1), (2), ... , (I) referem-se aos I níveis do fator A aninhado, na combinação dos níveis $j$ do fator $\mathrm{B}$ com o nível $k$ do fator $\mathrm{C}$.

\section{RESULTADOS E DISCUSSÃO}

Os dados que seguem (Tabela 1) referem-se à produção de matéria seca por planta de milho, g/vaso, cultivada por 35 dias em amostras de Latossolo Vermelho-Amarelo, de um experimento em esquema fatorial envolvendo quatro níveis de fontes de fósforo, dois níveis para modo de preparo e dois níveis para modo de aplicação, no delineamento inteiramente ao acaso, onde todos os fatores foram considerados de efeitos fixos.

O modelo matemático adotado é o mesmo citado na seção anterior. A principal hipótese a ser testada é referente à interação de fonte, modo de preparo e modo de aplicação, dada em $\mathrm{H}_{01}$. O quadro da análise da variância, com o teste $F$, é dado a seguir (Quadro 1). O programa SAS elaborado para esta análise encontra-se no fim deste item.

Pelo valor de $\mathrm{F}$ obtido na análise da variância observa-se que a interação de F x P x M foi significativa, podendo indicar a existência de um efeito diferenciado de fontes de fosfato $(F)$, dada cada combinação dos níveis de modo de preparo (P) com os níveis de modo de aplicação (M). Dando seqüência à análise estatística, o procedimento aplicado consiste na decomposição da soma dos graus de liberdade da

\begin{tabular}{|c|c|c|c|c|c|c|c|}
\hline \multirow[t]{2}{*}{ Fonte } & \multirow{2}{*}{$\begin{array}{l}\text { Preparo } \\
\text { Original }\end{array}$} & \multirow{2}{*}{$\begin{array}{l}\text { M. aplic } \\
\text { Vol. total }\end{array}$} & \multicolumn{4}{|c|}{ Repetições } & \multirow{2}{*}{$\begin{array}{l}\text { Média } \\
11,4\end{array}$} \\
\hline & & & 12,3 & 10,8 & 11,4 & 11,1 & \\
\hline \multirow{3}{*}{ FA1 } & & $1 \%$ vol. & 18,6 & 17,8 & 15,7 & 16,1 & 17,1 \\
\hline & Lavado & Vol. total & 8,0 & 7,7 & 7,5 & 6,7 & 7,5 \\
\hline & & $1 \%$ vol. & 4,8 & 5,7 & 5,3 & 5,7 & 5,4 \\
\hline \multirow{4}{*}{ FA2 } & Original & Vol. total & 9,0 & 9,7 & 9,1 & 8,3 & 9,0 \\
\hline & & $1 \%$ vol. & 14,8 & 13,2 & 13,8 & 14,1 & 14,0 \\
\hline & Lavado & Vol. total & 6,1 & 6,6 & 6,8 & 6,5 & 6,5 \\
\hline & & $1 \%$ vol. & 5,4 & 6,1 & 6,2 & 6,2 & 6,0 \\
\hline \multirow{4}{*}{ FA3 } & Original & Vol. total & 7,7 & 9,7 & 8,4 & 8,3 & 8,5 \\
\hline & & $1 \%$ vol. & 12,6 & 13,4 & 15,0 & 12,8 & 13,5 \\
\hline & Lavado & Vol. total & 6,8 & 7,6 & 8,1 & 6,8 & 7,3 \\
\hline & & $1 \%$ vol. & 5,5 & 8,3 & 8,9 & 7,7 & 7,6 \\
\hline \multirow{4}{*}{ FA4 } & Original & Vol. total & 7,0 & 7,7 & 9,5 & 7,4 & 7,9 \\
\hline & & $1 \%$ vol. & 6,3 & 6,3 & 6,7 & 7,4 & 6,7 \\
\hline & Lavado & Vol. total & 7,9 & 7,6 & 7,4 & 7,0 & 7,5 \\
\hline & & $1 \%$ vol. & 7,8 & 6,8 & 8,1 & 8,5 & 7,8 \\
\hline
\end{tabular}

Tabela 1. Dados de produção de matéria seca por planta de milho. 
Quadro 1. Análise da variância do experimento de produção de matéria seca.

\begin{tabular}{lccccc}
\hline C.V. & GL & SQ & QM & F & Prob > F \\
Fonte (F) & 3 & 66,941718 & 22,313906 & 34,08 & 0,0001 \\
Preparo (P) & 1 & 263,656406 & 263,656406 & 402,69 & 0,0001 \\
Fx P & 3 & 140,671718 & 46,890572 & 71,62 & 0,0001 \\
M. aplic (M) & 1 & 37,668906 & 37,668906 & 57,53 & 0,0001 \\
Fx M & 3 & 22,364218 & 7,454739 & 11,39 & 0,0001 \\
MxP & 1 & 66,626406 & 66,626406 & 101,76 & 0,0001 \\
FxPxM & 3 & 47,436718 & 15,812239 & 24,15 & 0,0001 \\
Resíduo & 48 & 31,4275 & 0,654739 & & \\
\hline Total & 63 & 676,793593 & & & \\
\hline
\end{tabular}

interação $\mathrm{F} \times \mathrm{P} \times \mathrm{M}$ com os graus de liberdade de fonte de fosfato $(F)$, os graus de liberdade da interação $F x$ $\mathrm{P}$ e os graus de liberdade de $\mathrm{F} \times \mathrm{M}$, isto é: três graus de liberdade para interação $\mathrm{F} \times \mathrm{P} \times \mathrm{M}$, mais os três graus de liberdade de $\mathrm{F}$, mais os três graus de liberdade da interação $\mathrm{F} \times \mathrm{P}$ e mais os três graus de liberdade da interação $\mathrm{F} \times \mathrm{M}$, totalizando 12 graus de liberdade. Desse modo temos que: 3 graus de liberdade para o efeito de F, dado o nível 1 de $P$ com o nível 1 de $\mathrm{M} ; 3$ graus de liberdade para efeito de $\mathrm{F}$, dado o nível 1 de $\mathrm{P}$ com o nível 2 de $\mathrm{M} ; 3$ graus de liberdade para o efeito de $\mathrm{F}$, dado o nível 2 de $\mathrm{P}$ com o nível 1 de $\mathrm{M}$, e 3 graus de liberdade para o efeito de $\mathrm{F}$, dado o nível 2 de $\mathrm{P}$ com o nível 2 de $\mathrm{M}$.

A hipótese de interesse a ser testada é:

$\mathrm{H}_{05}: \mu_{1(\mathrm{jk})}=\mu 2_{(\mathrm{jk})}==\mu_{\mathrm{I}(\mathrm{jk})}$ vs. $\mathrm{H}_{\mathrm{a} 5}$ : pelo menos

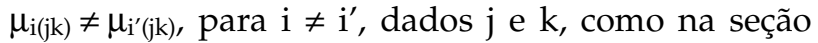
anterior, sendo: $\mathrm{i}=1, \ldots, 4$ (níveis de fonte de fosfato), $j=1,2$ (modo de preparo) e $k=1,2$ ( modo de aplicação). Os resultados obtidos encontram-se no quadro da análise da variância, a seguir (Quadro 2).
Para ilustrar a obtenção dos resultados da decomposição da interação através da utilização do comando CONTRAST do PROC GLM do SAS, considera-se o efeito de fonte de $\mathrm{P}$, dado o nível 1 do modo de preparo com o nível 1 do modo de aplicação, com 3 graus de liberdade, podendo ser fracionado em três contrastes ortogonais, com 1 grau de liberdade para cada um, cuja hipótese a ser testada é:

$$
\begin{gathered}
\mathrm{H}_{06}: \mathrm{Y}\left(\mathrm{h}_{\mathrm{jk}}\right)=0 \text { vs. } \mathrm{H}_{\mathrm{a} 6}: \mathrm{Y}\left(\mathrm{h}_{\mathrm{jk}}\right) \neq 0, \text { para } h=1,2,3 ; \\
j=1,2 \text { e } k=1,2 \text {, onde } \mathrm{Y}\left(\mathrm{h}_{\mathrm{jk}}\right)=\sum_{\mathrm{i}=1}^{4} c_{h i} \mu_{\mathrm{i}(\mathrm{jk})} \operatorname{com} \sum_{\mathrm{i}=1}^{4} c_{h i}=0, \mathrm{e} \\
\mathrm{Y}\left(\mathrm{h}_{\mathrm{jk}}\right)=\sum_{\mathrm{i}=1}^{4} \mathrm{chi}_{\mathrm{i}} \alpha_{\mathrm{i}}+\sum_{\mathrm{i}=1}^{4} \mathrm{chi}_{\mathrm{i}}(\alpha \beta)_{\mathrm{i}(\mathrm{j})}+\sum_{\mathrm{i}=1}^{4} \mathrm{chi}_{\mathrm{i}}(\alpha \delta)_{\mathrm{i}(\mathrm{k})}+ \\
+\sum_{\mathrm{i}=1}^{4} \mathrm{chi}_{\mathrm{i}}(\alpha \beta \delta)_{\mathrm{i}(\mathrm{jk})}
\end{gathered}
$$

Assim, em termos de programação $S A S$, tem-se:

CONTRAST "Y1-F(P1xM1)" F 111 -3 F*P 101010 -3 0 F*M 101010 -3 0 $\mathrm{F}^{*} \mathrm{P}^{*} \mathrm{M} 100001000010000-3000$;

CONTRAST "Y2-F(P1xM1)" F 11 - -20 F*P $1010-20000 F^{*}$ M $1010-2000$ $F^{*} P^{*} \mathrm{M} 1000010000-2000000000$;

CONTRAST "Y3-F(P1xM1)" F $1-100$ F*P $10-1000000$ F*M 10 - 1000000 $F^{*} P^{*} \mathrm{M} 10000-10000000000000$;

CONTRAST "F(P1xM1)" F 11 1-3 F*P 101010 -3 0 F*M 101010 -30

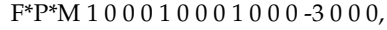

$\mathrm{F} 11-20 \mathrm{~F}^{*} \mathrm{P} 1010-2000 \mathrm{~F}^{*} \mathrm{M} 1010-2000$
$\mathrm{~F}^{*} \mathrm{P} * \mathrm{M} 10001000-20000000$

F 1 - $100 \mathrm{~F}^{*} \mathrm{P} 10-100000 \mathrm{~F}^{*} \mathrm{M} 10-100000$ $F^{*} P^{*} M 10000-1000000000000000$.

A seguir é mostrado o resultado do $S A S$, referente ao segmento de programação utilizando o comando CONTRAST do PROC GLM, conforme ilustrado anteriormente.

Quadro 2. Decomposição da interação tripla do fator fontes de P aninhado com a interação dupla Modo de Aplicação vs. Preparo.

\begin{tabular}{lcrrrc}
\hline C.V. & GL & \multicolumn{1}{c}{ SQ } & \multicolumn{1}{c}{ QM } & \multicolumn{1}{c}{ F } & Prob > F \\
Preparo (P) & 1 & 263,656406 & 263,656406 & 402,69 & 0,0001 \\
M. aplic. (M) & 1 & 37,6689062 & 37,6689062 & 57,53 & 0,0001 \\
M x P & 1 & 66,626406 & 66,626406 & 101,76 & 0,0001 \\
F(P1 x M1) & 3 & 28,062500 & 9,354167 & 14,28 & 0,0001 \\
F(P1 x M2) & 3 & 229,5225 & 76,5075 & 116,90 & 0,0001 \\
F(P2 x M1) & 3 & 2,626875 & 0,875625 & 1,34 & 0,2733 \\
F(P2 x M2) & 3 & 17,2025 & 5,734167 & 8,76 & 0,0001 \\
F(P x M) & 12 & 277,414375 & & & \\
Resíduo & 48 & 31,4275 & 0,654739 & & \\
\hline Total & 63 & 676,793593 & & \\
\hline
\end{tabular}




\begin{tabular}{ccrrrr}
\hline Contrast & DF & \multicolumn{1}{c}{ SQ } & \multicolumn{1}{c}{ MS } & \multicolumn{1}{c}{ F } & Pr $>$ F \\
\hline Y1-f(p1xm1) & $\mathbf{1}$ & 9.18750000 & 9.18750000 & 14.03 & $\mathbf{0 . 0 0 0 5}$ \\
Y2-f(p1xm1) & $\mathbf{1}$ & 7.59375000 & 7.59375000 & 11.60 & $\mathbf{0 . 0 0 1 3}$ \\
Y3-f(p1xm1) & $\mathbf{1}$ & 11.28125000 & 11.28125000 & 17.23 & $\mathbf{0 . 0 0 0 1}$ \\
f(p1xm1) & $\mathbf{3}$ & $\mathbf{2 8 . 0 6 2 5 0 0 0 0}$ & 9.35416667 & $\mathbf{1 4 . 2 9}$ & $\mathbf{0 . 0 0 0 1}$ \\
Y1-f(p1xm2) & $\mathbf{1}$ & 199.26750000 & 199.26750000 & 304.35 & $\mathbf{0 . 0 0 0 1}$ \\
Y2-f(p1xm2) & $\mathbf{1}$ & 11.34375000 & 11.34375000 & 17.33 & $\mathbf{0 . 0 0 0 1}$ \\
Y3-f(p1xm2) & $\mathbf{1}$ & 18.91125000 & 18.91125000 & 28.88 & $\mathbf{0 . 0 0 0 1}$ \\
f(p1xm2) & $\mathbf{3}$ & 229.52250000 & 76.50750000 & $\mathbf{1 1 6 . 8 5}$ & $\mathbf{0 . 0 0 0 1}$ \\
Y1-f(p2xm1) & $\mathbf{1}$ & 0.42187500 & 0.42187500 & 0.64 & $\mathbf{0 . 4 2 6 1}$ \\
Y2-f(p2xm1) & $\mathbf{1}$ & 0.30375000 & 0.30375000 & 0.46 & $\mathbf{0 . 4 9 9 1}$ \\
Y3-f(p2xm1) & $\mathbf{1}$ & 1.90125000 & 1.90125000 & 2.90 & $\mathbf{0 . 0 9 4 8}$ \\
f(p2xm1) & $\mathbf{3}$ & 2.62687500 & 0.87562500 & $\mathbf{1 . 3 4}$ & $\mathbf{0 . 2 7 3 3}$ \\
Y1-f(p2xm2) & $\mathbf{1}$ & 6.60083333 & 6.60083333 & 10.08 & $\mathbf{0 . 0 0 2 6}$ \\
Y2-f(p2xm2) & $\mathbf{1}$ & 9.88166667 & 9.88166667 & 15.09 & $\mathbf{0 . 0 0 0 3}$ \\
Y3-f(p2xm2) & $\mathbf{1}$ & 0.72000000 & 0.72000000 & 1.10 & $\mathbf{0 . 2 9 9 6}$ \\
f(p2xm2) & $\mathbf{3}$ & 17.20250000 & 5.73416667 & $\mathbf{8 . 7 6}$ & $\mathbf{0 . 0 0 0 1}$ \\
\hline
\end{tabular}

Outra decomposição pode ser feita, para outros fatores, de acordo com o interesse do pesquisador.

Conforme apresentado, o procedimento de análise estatística de um experimento com três fatores com interação significativa recai na decomposição dos graus de liberdade da interação nos efeitos aninhados dos fatores envolvidos. Esse tipo de procedimento não é obtido facilmente pelos softwares disponíveis, exigindo, invariavelmente, uma programação específica para a obtenção dos resultados desejados.

No módulo STAT do software $S A S$, isso é diferente, pois utiliza-se o comando CONTRAST do PROC GLM, para se obter o efeito aninhado testado corretamente. Apesar disso, é necessário certo conhecimento dos conceitos básicos de estatística experimental, para que se possa fazer tal decomposição de maneira correta, a fim de se obterem os resultados convenientes para a análise.

\section{APENNDICE: Programa SAS}

Programa SAS para Decomposição da Interação Tripla Significativa

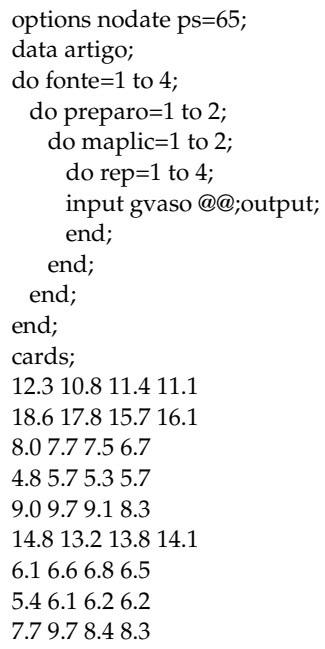

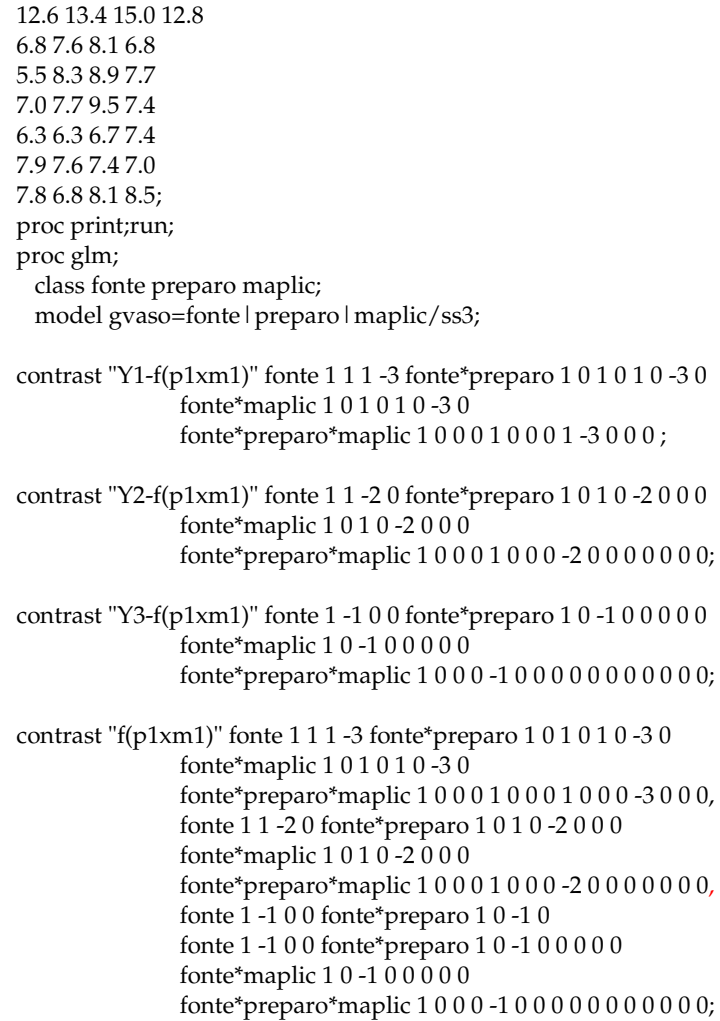

contrast "Y1-f(p1xm2)" fonte $111-3$ fonte*preparo $101010-30$ fonte*maplic $010101010-3$

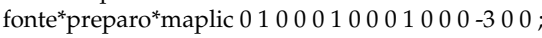

contrast "Y2-f(p1xm2)" fonte 11 -2 0 fonte* preparo $1010-2000$ fonte*maplic $01010-200$ fonte*preparo*maplic $010001000-20000000$;

contrast "Y3-f(p1xm2)" fonte $1-100$ fonte $^{*}$ preparo $10-100000$ fonte ${ }^{*}$ maplic $010-100000$ fonte* preparo*maplic $01000-10000000000$;

contrast "f(p1xm2)" fonte $111-3$ fonte* preparo $101010-30$ fonte*maplic $010101010-3$ fonte* preparo*maplic $0100010001000-300$ fonte $11-20$ fonte*preparo $1010-2000$ fonte*maplic $010010-2000$ fonte*preparo*maplic $010001000-2000000$, fonte $1-100$ fonte $^{*}$ preparo $10-100000$ fonte*maplic $010-100000$

fonte* preparo*maplic $01000-100000000000$;

contrast "Y1-f(p2xm1)" fonte $111-3$ fonte* preparo 0101010 -3 fonte*maplic $10101010-30$ fonte ${ }^{*}$ preparo*maplic $00100010001000-30$;

contrast "Y2-f(p2xm1)" fonte 11 -2 0 fonte $^{*}$ preparo $01010-200$ fonte*maplic $10010-20000$

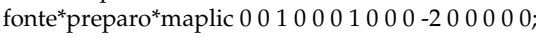

contrast "Y3-f(p2xm1)" fonte 1 -1 00 fonte*preparo $010-100000$ fonte*maplic $10-1000000$ fonte*preparo*maplic $001000-10000000000$;

contrast "f(p2xm1)" fonte $111-3$ fonte*preparo $0101010-3$ fonte*maplic $10101010-30$

fonte*preparo*maplic $00100010001000-30$, fonte 11 -2 0 fonte* preparo $01010-200$ fonte*maplic $100100-2000$ 
fonte* preparo*maplic $00100001000-2000000$, fonte $1-100$ fonte* preparo $010-10000$ fonte*maplic $10-1000000$

fonte*preparo*maplic $001000-10000000000$;

contrast "Y1-f(p2xm2)" fonte $111-3$ fonte*preparo $0101010-3$ fonte*maplic $010101010-3$

fonte $^{*}$ preparo*maplic $0001000100001000-3$;

contrast "Y2-f(p2xm2)" fonte 11 -2 0 fonte*preparo $01010-200$ fonte*maplic $01010-200$

fonte*preparo*maplic $00010001000-20000$;

contrast "Y3-f(p2xm2)" fonte $1-100$ fonte $^{*}$ preparo $010-10000$

fonte*maplic $010-100000$

fonte ${ }^{*}$ preparo*maplic $0001000-1000000000$;

contrast "f(p2xm2)" fonte $111-3$ fonte preparo 0101010 -3 fonte*maplic $010101010-3$

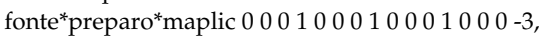
fonte $11-20$ fonte* preparo $01010-200$

fonte*maplic $0101010-200$

fonte* preparo*maplic $000100010000-200000$,

fonte $1-100$ fonte*preparo $010-10000$

fonte*maplic $010-100000$

run;

fonte ${ }^{*}$ preparo*maplic $0001000-1000000000$;

proc glm;

class fonte preparo maplic;

model gvaso=fonte I preparo I maplic/ss3;

lsmeans fonte ${ }^{*}$ preparo*maplic/slice=preparo*maplic; run;

\section{CONCLUSÕES}

1. Essas decomposições podem ser obtidas facilmente para níveis quantitativos e qualitativos através do software $S A S$ que, apesar de ser um software fechado, fornece algumas condições de programação para se obterem procedimentos de análise de dados que, manualmente, seriam complexos e trabalhosos.

2. Nas versões mais recentes do $S A S$, tais como 6.11 e 6.12, já existe a opção SLICE do comando LSMEANS do PROC GLM, a qual fornece resultados análogos aos obtidos com o uso do comando CONTRAST, e cuja sintaxe é a seguinte:

\section{LSMEANS $F^{*} P^{*} \mathrm{M} /$ SLICE $=\mathrm{P}^{*} \mathrm{M}$;}

Através da opção SLICE $=\mathrm{P}^{*} \mathrm{M}$, os níveis do fator modo de preparo combinados com os níveis do fator modo de aplicação são fixados, obtendo-se, assim, o efeito de fontes de fosfato para cada combinação dos níveis de modo de preparo com modo de aplicação.

\section{AGRADECIMENTOS}

Os autores agradecem ao Prof. Dr. Luiz Ignácio Prochnow, do Departamento de Solos e Nutrição de Plantas da ESALQ/USP, pelos dados fornecidos e utilizados para ilustrar os resultados obtidos na aplicação da metodologia apresentada, e, aos revisores, pelas sugestões que aprimoraram este trabalho.

\section{REFERÊNCIAS BIBLIOGRÁFICAS}

KUEHL, R.O. Statistical principles of research design and analysis. Belmont, California: Duxbury Press, 1994. 686 p.

FISHER, R.A. The design of experiments. Edinburg and London: Oliver and Boyd, 1935. 260p.

HINKELMANN, K.; KEMPTHORNE, O. Design and analysis of experiments: introduction to experimental design. New York, John Wiley \& Sons, 1994. v.1, 495 p.

NOGUEIRA, M.C.S. Estatística experimental aplicada à experimentação agronômica. Piracicaba: DME/ESALQ, 1997. $250 \mathrm{p}$.

SAS Institute. SAS/STAT. User's Guide. Version $6,4^{\text {th }}$ ed. Cary, NC, USA,1990. v.2, 846 p.

YATES, F. The design and analysis of factorial experiments. Commnwealth Bureau of Soil Science, 1937. 95 p. (Technical Communication, 35). 